\title{
MEDIDA DO ÂNGULO HORIZONTAL DE ATAQUE DOS DISCOS DE GRADES AGRÍCOLAS DE DUPLA AÇÃO E APLICAÇÃO A UMA PROPRIEDADE AGRÍCOLA $\left({ }^{1}\right)$
}

\author{
RUBISMAR STOLF $\left(\left(^{*}\right)\right.$; JAIR ROSAS DA SILVA $\left({ }^{3}\right)$; JORGE ALBERTO MONTOYA GOMEZ $\left({ }^{4}\right)$
}

\begin{abstract}
RESUMO
O conhecimento do ângulo de ataque de grades agrícolas é importante, pois o mesmo influencia a exigência em força de tração, a profundidade e a qualidade da gradagem. O objetivo deste trabalho foi desenvolver um método de medida do ângulo horizontal, de ataque, dos discos de grades de dupla ação com as seguintes características: rápido, de fácil medida e preciso, para aplicação no local de trabalho das grades. O princípio do método proposto baseia-se no fato de que é possível circunscrever grades de dupla ação em um trapézio isósceles e no teorema da trigonometria no qual ângulos com lados perpendiculares entre si formam ângulos iguais. Assim, o método desenvolvido pode ser enunciado como segue: "o seno do ângulo de ataque de uma grade de dupla ação é igual à diferença entre a base maior e a base menor dividida por duas vezes o lado do trapézio". Dessa forma, três medidas de comprimento realizadas com uma trena, ou seja, base maior, base menor e o lado do trapézio, permitem determinar o valor do seno e, consequentemente, o próprio ângulo de ataque da grade. Foram levantados os ângulos de ataque de onze grades agrícolas de propriedade da Destilaria Londra, de Itaí, estado de São Paulo, comprovando a rapidez e facilidade de utilização do método.
\end{abstract}

Palavras-chave: grade, ângulo de ataque, ângulo de corte, ângulo horizontal.

\section{ABSTRACT \\ METHOD FOR MEASURING THE HORIZONTAL GANG ANGLE OF DOUBLE-ACTION DISC HARROWS AND ITS APPLICATION IN THE FIELD}

The knowledge of the horizontal attack angle is important, considering its influence in terms of harrowing tractor power requirement, and depth and quality of soil tillage. The aim was to develop a fast, easy to apply in the field and precise method to measure the horizontal angle (attack angle) of double action harrows. The method is based on the fact that it is possible to circumscribe the harrows (of double action) to an isosceles trapezium. Besides, it is based on a theorem of the trigonometry in which angles with perpendicular sides between themselves itself form equal angles. Thus, the developed method can be stated as follows: "the sine of the angle of attack is equal to the difference between the biggest and the smallest basis divided by twice the side of the trapezium". The developed method can be applied using only one meter measuring tape. In other words, through three measures of length, of the biggest and of the smallest basis and of the side of the trapezium it is possible to calculate the sine and consequently the gang angle of the harrow. The method was applied in all eleven harrows of Londra distillery enterprise, in Itai, Sao Paulo State, Brazil, proving its easy application.

Key words: harrow, disc, gang angle, weep angle, horizontal angle

$\left.{ }^{1}\right)$ Trabalho apresentado no XXXVI Congresso Brasileiro de Engenharia Agrícola, 30 de julho a 02 de agosto de 2007 Bonito (MS). Recebido para publicação em 15 de setembro de 2008 e aceito para publicação em 23 de outubro de 2009.

$\left(^{2}\right)$ Departamento de Recursos Naturais e Proteção Ambiental, Universidade Federal de São Carlos, Campus Araras. Via Anhanguera, km 174, Caixa Postal 153, 13600-970 Araras (SP). E-mail: rubismar@cca.ufscar.br. $\left(^{*}\right)$ Autor correspondente.

$\left({ }^{3}\right)$ Centro de Engenharia e Automação, Instituto Agronômico, APTA, Secretaria da Agricultura e Abastecimento do Estado de São Paulo. Rodovia Dom Gabriel Paulino Bueno Couto, km 65, Caixa Postal 26, 13201-970 Jundiaí (SP). E-mail: jairrosas@iac.sp.gov.br

$\left.{ }^{4}\right)$ Rua Barão de Piracicamirim, 889, 13416-335 Piracicaba (SP). E-mail: jorge_montoya_gomez@yahoo.com.br 


\section{INTRODUÇÃO}

As grades agrícolas constituem uma classe de implementos dos mais utilizados para mobilização do solo em preparo para o plantio. Em cana-de-açúcar, estima-se no Brasil que um milhão de hectares por ano é mobilizado para a reforma de canaviais, com a utilização de grades. Na cultura do milho nove milhões foram plantados sob plantio convencional na safra 20042005 (FNP, 2006). Considerando três gradagens por unidade de área, o somatório do percurso das grades em áreas de cultura do milho, em um ano, no Brasil, equivale aproximadamente a 2700 voltas em torno da Terra, demonstrando-se assim a importância das grades agrícolas nos sistema de preparo do solo.

As grades agrícolas de discos são classificadas como de simples ou dupla ação, conforme tenham uma ou duas seções de discos trabalhando o solo (GALETI, 1981). Por meio de um levantamento realizado em quatro fabricantes de expressão nacional, verificou-se que apenas oito eram de ação simples e 431 de dupla ação e que as características dimensionais correlacionam-se com a potência recomendada pelos fabricantes (STOLF et al., 1981; STOLF \& Silva, 1996 a, b).

O ângulo de ataque das grades é definido como o ângulo horizontal formado entre o plano que passa pela circunferência do disco e a direção do movimento (O'DOGHERTY et al., 1996; KeLLER, 2004). Quanto maior for o ângulo maior será a profundidade de trabalho, maior a exigência em força de tração, maior deslocamento lateral da leiva (STOLF, 1986b), efeito detectado também nos arados (Mello, 2004). Portanto, esse conhecimento pode ser utilizado tanto para melhorar a qualidade do serviço como para reduzir a força de tração, adequando melhor o sistema trator-grade.

Não tendo sido detectado na literatura nenhum procedimento para a determinação do ângulo de ataque de grades agrícolas, o objetivo do estudo foi o de propor e testar um método rápido, de fácil medida e preciso, para uso no campo, em área de trabalho das grades.

\section{MATERIAL E MÉTODOS} premissas:

O método proposto baseia-se nas seguintes

a) É possível circunscrever a estrutura de grades de dupla ação em um trapézio isósceles;

b) Os ângulos com lados perpendiculares entre si formam ângulos iguais (teorema da trigonometria, Figura 1).

Com base nesses elementos da geometria e trigonometria e, medindo-se a base maior, a base menor e o lado do trapézio circunscrito, torna-se

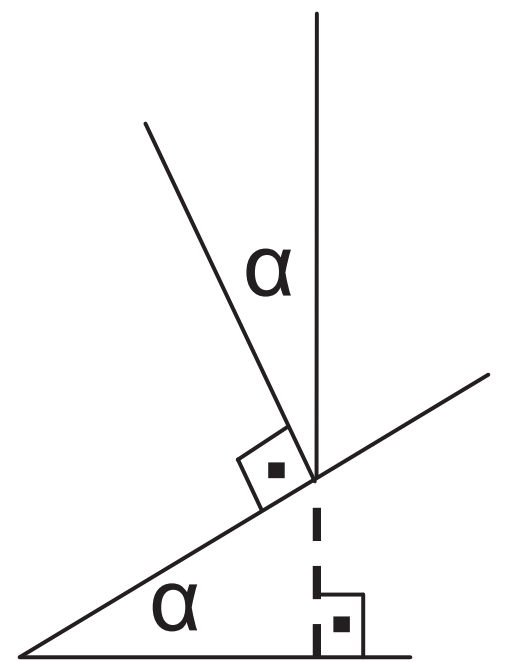

Figura 1. Ilustração do princípio trigonométrico aplicado na figura 2 para desenvolver o método: "ângulos com lados perpendiculares entre si formam ângulos iguais".

possível determinar o seno e conseqüentemente o ângulo de ataque de qualquer grade de discos de dupla ação. A figura 2 representa uma ilustração do formato trapezoidal da estrutura da grade, de lados iguais (L) e com as bases, maior e menor, representadas pelos segmentos $\mathrm{BM}$ e $\mathrm{Bm}$, respectivamente, paralelos entre si.

Pela trigonometria, tem-se que o seno do ângulo de ataque da grade de discos, também chamado de ângulo horizontal, ou de corte, é dado pela expressão:

$$
\operatorname{Sen}(\alpha)=(B M-B m) /(2 L)
$$

em que: $\alpha=$ ângulo de ataque ou de corte; $B M=$ base maior do trapézio isósceles; $\mathrm{Bm}=$ base menor do trapézio isósceles; $\mathrm{L}=$ lado do trapézio isósceles.

Por meio da aplicação da fórmula da função trigonométrica inversa:

$$
y=\operatorname{arcsen} x
$$

determina-se o ângulo horizontal de corte da grade $(\alpha)$.

Para maior facilidade e praticidade de aplicação do método proposto, gerou-se uma tabela de conversão do seno para o valor do ângulo horizontal de corte, conforme a tabela 1. Dessa forma, dispensa-se o uso da função trigonométrica inversa e transformações de radianos em graus, que possam dificultar o cálculo, em condições de campo. Os pontos referenciais recomendados para proceder as medidas são os extremos das barras dianteiras e traseiras de suporte dos discos, ou seja, as arestas, garantindo simetria entre os pontos escolhidos, conforme se observa na figura 2. 
Tabela 1. Tabela de obtenção do ângulo de ataque da grade a partir de seu seno

\begin{tabular}{cc|cc|cc|cc|cc|ccc|ccc}
\hline $\operatorname{Sen} \alpha$ & $\alpha$ & $\operatorname{Sen} \alpha$ & $\alpha$ & $\operatorname{Sen} \alpha$ & $\alpha$ & $\operatorname{Sen} \alpha$ & $\alpha$ & $\operatorname{Sen} \alpha$ & $\alpha$ & $\operatorname{Sen} \alpha$ & $\alpha$ & $\operatorname{Sen} \alpha$ & $\alpha$ & $\operatorname{Sen} \alpha$ & $\alpha$ \\
\hline 0,259 & $15,0^{\circ}$ & 0,282 & $16,4^{\circ}$ & 0,306 & $17,8^{\circ}$ & 0,329 & $19,2^{\circ}$ & 0,352 & $20,6^{\circ}$ & 0,375 & $22,0^{\circ}$ & 0,397 & $23,4^{\circ}$ & 0,419 & $24,8^{\circ}$ \\
0,262 & $15,2^{\circ}$ & 0,286 & $16,6^{\circ}$ & 0,309 & $18,0^{\circ}$ & 0,332 & $19,4^{\circ}$ & 0,355 & $20,8^{\circ}$ & 0,378 & $22,2^{\circ}$ & 0,400 & $23,6^{\circ}$ & 0,423 & $25,0^{\circ}$ \\
0,266 & $15,4^{\circ}$ & 0,289 & $16,8^{\circ}$ & 0,312 & $18,2^{\circ}$ & 0,335 & $19,6^{\circ}$ & 0,358 & $21,0^{\circ}$ & 0,381 & $22,4^{\circ}$ & 0,404 & $23,8^{\circ}$ & 0,426 & $25,2^{\circ}$ \\
0,269 & $15,6^{\circ}$ & 0,292 & $17,0^{\circ}$ & 0,316 & $18,4^{\circ}$ & 0,339 & $19,8^{\circ}$ & 0,362 & $21,2^{\circ}$ & 0,384 & $22,6^{\circ}$ & 0,407 & $24,0^{\circ}$ & 0,429 & $25,4^{\circ}$ \\
0,272 & $15,8^{\circ}$ & 0,296 & $17,2^{\circ}$ & 0,319 & $18,6^{\circ}$ & 0,342 & $20,0^{\circ}$ & 0,365 & $21,4^{\circ}$ & 0,388 & $22,8^{\circ}$ & 0,41 & $24,2^{\circ}$ & 0,432 & $25,6^{\circ}$ \\
0,276 & $16,0^{\circ}$ & 0,299 & $17,4^{\circ}$ & 0,322 & $18,8^{\circ}$ & 0,345 & $20,2^{\circ}$ & 0,368 & $21,6^{\circ}$ & 0,391 & $23,0^{\circ}$ & 0,413 & $24,4^{\circ}$ & 0,435 & $25,8^{\circ}$ \\
0,279 & $16,2^{\circ}$ & 0,302 & $17,6^{\circ}$ & 0,326 & $19,0^{\circ}$ & 0,349 & $20,4^{\circ}$ & 0,371 & $21,8^{\circ}$ & 0,394 & $23,2^{\circ}$ & 0,416 & $24,6^{\circ}$ & 0,438 & $26,0^{\circ}$ \\
\hline
\end{tabular}
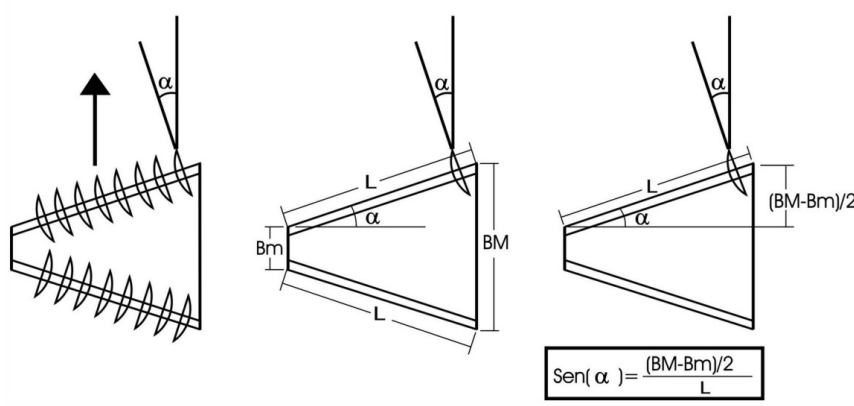

Figura 2. (a) Esquema de uma grade e o ângulo de ataque dos discos. (b) Medidas de comprimento: BM, Bm e L para a determinação do seno do ângulo de ataque. (c) Triângulo ilustrando que $\operatorname{sen}(\alpha)=(B M-B m) /(2 \mathrm{~L})$.

Para o levantamento de dados de campo foram consideradas todas as grades de discos empregadas no preparo de solo das áreas agrícolas de produção de cana-de-açúcar administradas pela Destilaria Londra, situadas no município de Itaí, Estado de São Paulo, Brasil. Quanto ao porte e à potência exigida, as grades ensaiadas foram agrupadas nas seguintes classes: leve, média ou pesada, de acordo com STOLF (1986a), discriminando-se suas marcas e modelos. O ângulo de ataque de cada grade de discos foi levantado conforme o procedimento proposto.

As leituras foram realizadas por duas pessoas utilizando uma trena metálica retrátil de três metros.

\section{RESULTADOS E DISCUSSÃO}

A aplicação do método e suas restrições são representadas didaticamente através do seguinte exemplo: desejando-se determinar o ângulo de ataque de uma grade de discos de dupla ação, dirigiu-se até a área de trabalho em que se operava um espécime do referido implemento de preparo secundário do solo, onde foi efetuada a determinação das medidas da base maior, base menor e lado do trapézio imaginário a ela circunscrito, obtendo-se as seguintes medidas: $\mathrm{BM}=3,86 \mathrm{~m}$; $\mathrm{Bm}=2,06 \mathrm{~m}$ e $\mathrm{L}=2,69 \mathrm{~m}$. De posse desses elementos, calcular: a) o ângulo de ataque e b) o ângulo de "trava" da grade, conforme segue:
Substituindo-se os valores determinados na grade de discos em serviço na Equação (1), calculase primeiramente o seno do ângulo de corte:

$$
\begin{aligned}
\operatorname{sen}(\alpha) & =(3,86-2,06) /(2 \times 2,69) \\
\operatorname{sen}(\alpha) & =0,335
\end{aligned}
$$

Por meio da aplicação da fórmula da função inversa (Equação 2) ou utilizando os valores apresentados na tabela 1, destinada a simplificar o cálculo, verifica-se que o ângulo, cujo seno vale 0,335 , é de 19,6 graus. Resposta: a) ângulo de ataque $=19,6$ graus $\left(19^{\circ} 36^{\prime}\right)$. b) ângulo de trava, o dobro $=39,2$ graus $\left(39^{\circ} 12^{\prime}\right)$. Faz-se necessário observar o seguinte fato: o valor de 39,2 graus representa realmente a estimativa do ângulo de trava. Porém, caso o conjunto dianteiro e traseiro sejam operados com ângulos diferentes, o ângulo obtido, $19^{\circ} 30^{\prime}$ representará o valor médio dos ângulos de ataque dos discos dianteiros e traseiros. Serão exatamente iguais se os corpos de ação forem regulados com simetria em relação à perpendicular ao movimento, o que é tecnicamente recomendado.

Na tabela 2 é apresentado o levantamento das grades da propriedade agrícola mencionada. A primeira discussão se refere à validação do método, comprovando-se a facilidade de aplicação e rapidez na realização das três leituras, efetuadas em cerca de nove minutos. Quanto à precisão do método, a teoria do formato trapezoidal foi confirmada experimentalmente na avaliação de onze grades. As possíveis fontes de erro que possam ocorrer no emprego do método se referem às medidas de comprimento mencionadas. No exemplo apresentado, é possível realizar simulações verificando que apenas erros grosseiros, de leitura, resultariam em diferenças sensíveis na determinação do ângulo desejado.

A segunda questão a ser discutida é se os ângulos das grades estão regulados de forma correta. Consultando os catálogos de três empresas fabricantes nacionais, não foram constatadas recomendações quanto à regulagem do ângulo de ataque. Consultandose o departamento de engenharia da empresa Marchesan, foram obtidas as seguintes informações: há três pontos de regulagem correspondentes a 17,5 graus, 20,0 graus e 22,5 graus (respectivamente ângulos de trava de 35 graus, 40 graus e 45 graus). 
Tabela 2. Cálculo do ângulo de ataque das grades da Destilaria Londra, Itaí, Estado São Paulo

\begin{tabular}{|c|c|c|c|c|c|c|c|c|}
\hline $\begin{array}{l}\text { Classes de grade } \\
\text { segundo STOLF (1986 a) }\end{array}$ & No. & Marca & Modelo & $\begin{array}{l}\text { Base maior } \\
\text { BM }\end{array}$ & $\begin{array}{l}\text { Base menor } \\
\qquad \mathrm{Bm}\end{array}$ & $\begin{array}{l}\text { Lado } \\
\qquad \mathrm{L}\end{array}$ & $\begin{array}{c}\operatorname{Sen}(\alpha)= \\
(\mathrm{BM}-\mathrm{Bm}) /(2 \mathrm{~L})\end{array}$ & $\begin{array}{l}\text { Ângulo de } \\
\text { ataque } \alpha\end{array}$ \\
\hline & & & & & $-\mathrm{m}-$ & 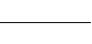 & & \\
\hline \multirow{5}{*}{$\begin{array}{l}\text { Grade pesada/ } \\
\text { aradora }\end{array}$} & 1 & Marchesan & GAPCR & 3,86 & 2,06 & 2,69 & 0,335 & 19,6 \\
\hline & 2 & Marchesan & GAPCR & 3,87 & 2,06 & 2,69 & 0,336 & 19,7 \\
\hline & 3 & Marchesan & GAPCR & 3,86 & 2,06 & 2,71 & 0,332 & 19,4 \\
\hline & 4 & Marchesan & GAPCR & 3,85 & 2,06 & 2,70 & 0,331 & 19,4 \\
\hline & 5 & Marchesan & GAPCR & 3,88 & 2,08 & 2,71 & 0,332 & 19,4 \\
\hline Média / & 1 & Marchesan & GAICR & 3,85 & 1,47 & 3,54 & 0,336 & 19,6 \\
\hline \multirow[t]{4}{*}{ intermediária } & 2 & Marchesan & GAICR & 3,85 & 1,45 & 3,55 & 0,338 & 19,8 \\
\hline & 3 & Marchesan & GAICR & 3,85 & 1,46 & 3,55 & 0,337 & 19,7 \\
\hline & 4 & Piccin & GAICR & 3,96 & 1,69 & 3,46 & 0,328 & 19,1 \\
\hline & 5 & Piccin & GAICR & 3,97 & 1,70 & 3,46 & 0,328 & 19,1 \\
\hline Grade leve & 1 & Civemasa & SAC & 4,62 & 2,16 & 4,28 & 0,287 & 16,7 \\
\hline
\end{tabular}

GAPCR: grade aradora pesada com Controle Remoto; GAICR: grade aradora intermediária com controle remoto; CR: Controle remoto para a roda de transporte. SAC: grade aradora leve

O primeiro valor é utilizado para grades niveladoras, enquanto o segundo ou o terceiro ponto de regulagem para grades aradoras, tanto intermediárias como pesadas, identificadas comercialmente pelas siglas GAICR e GAPCR.

Assim, é possível discutir que, independentemente das marcas comerciais das grades, seja Marchesan, Piccin ou Civemasa, as mesmas possuem ângulos de ataque regulados corretamente, ou seja, a grade niveladora com um valor menor que permite ser operada em maior velocidade e mais na superfície do solo e as grades intermediárias e pesadas, reguladas para um valor intermediário próximo de 20 graus (Tabela 2). Caso seja necessário promover maior penetração no solo pelas grades aradoras, haveria ainda a opção do terceiro ponto (22,5 graus). Por outro lado, para solos com alto teor da fração areia, haveria a opção de regulagem no primeiro ponto $(17,5$ graus) na operação das grades aradoras.

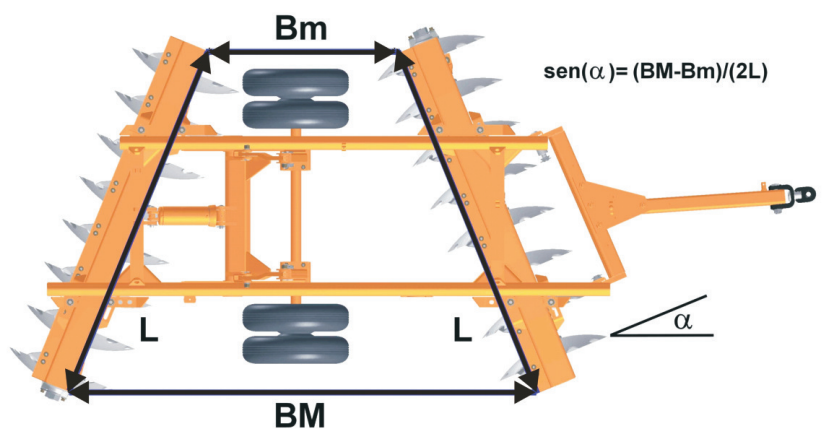

Figura 3. Exemplo de aplicação do método em uma grade pesada MARCHESAN modelo GAPCR 16 discos (grade aradora pesada controle remoto).
Na figura 3, visualiza-se um exemplo de tomada de medidas das bases e lado do trapézio circunscrito para aplicação do método em uma grade de dupla ação grade "offset" (seções deslocadas entre si) da marca comercial Marchesan e, na Figura 4, é apresentada a mesma tomada de medidas para a aplicação do método em uma grade de dupla ação em "tandem" (seções conjugadas).

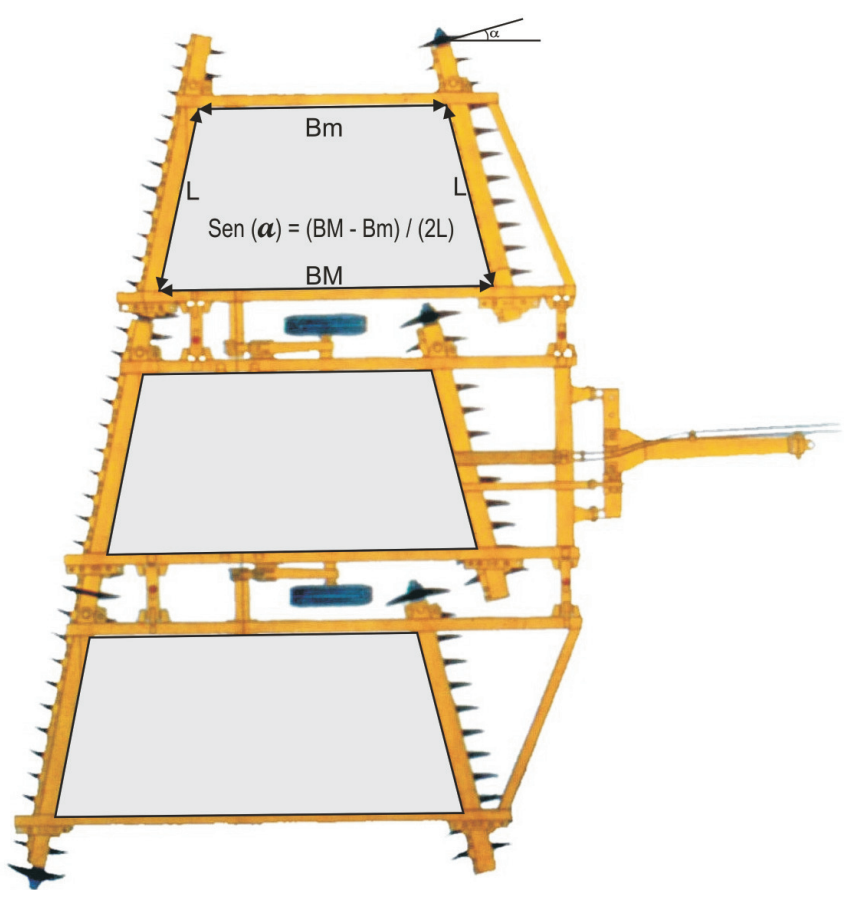

Figura 4. Grade em "tandem" de três conjuntos. Estando os três conjuntos com a mesma regulagem de ângulo de ataque, basta aplicar o método em um conjunto. 


\section{CONCLUSÃO}

Com o objetivo de proporcionar praticidade e facilidade no levantamento de um importante parâmetro operacional de grades agrícolas, desenvolveu-se um método prático, simples e expedito destinado à determinação do ângulo horizontal de ataque de grades agrícolas de discos de dupla ação, podendo o mesmo ser aplicado no próprio local no qual a grade está operando, sem necessidade de condições ou equipamentos especiais.

\section{AGRADECIMENTOS}

Ao Diretor Enzo Mazzonetto Valler, da Destilaria Londra, pela oportunidade, e ao funcionário Clayton Ferreira dos Santos pelo apoio na determinação das medidas de campo.

\section{REFERÊNCIAS}

FNP. Anuário da pecuária brasileira. São Paulo: FNP Consultoria \& Comércio, 2006. 370 p.

GALETI, P. A. Mecanização agrícola: preparo do solo. Campinas: Instituto Campineiro de Ensino Agrícola, 1981. $220 \mathrm{p}$.

KELLER T. Soil compaction and soil tillage: studies in agricultural soil mechanics. 2004. 75f. Tese (Doctor). Department of Soil Sciences, Swedish University of Agricultural Sciences, Uppsala.

MELLO, R.C. Alterações físicas em um Latossolo Roxo em função da velocidade de aração e do ângulo horizontal dos discos. Acta Scientiarum Agronomy, v.26, p.35-40, 2004.

O’DOGHERTY, M.J., GODWIN, R.J., HANN, M.J., ALGHAZAL, A.A. A geometrical analysis of inclined and tilted spherical plough discs. Journal of Agricultural Engineering Research, v.63, p.205-218, 1996.

STOLF, R.; FERNANDES, J.; FURLANI NETO, V. L. Uma análise da equivalência entre as potências de tratores de pneus e de esteiras e suas correlações com características de grade, através das recomendações dos fabricantes. In: CONGRESSO NACIONAL DA STAB, 2, 1981, Rio de Janeiro. Anais... Rio de Janeiro: STAB, 1981. v.3/4. p.395-414. Reeditado: Brasil Açucareiro, v.103, p.28-34, 1985.

STOLF, R. Grade leve, média, pesada e super pesada: classificação e função. Álcool \& Açúcar, v.6, p.3644, 1986a.

STOLF, R. Grades e seus tratores: exemplos práticos de seleção. Álcool \& Açúcar, v.6, p.62-8, 1986b.

STOLF, R.; SILVA, J. J. Características de quatrocentos e trinta e um modelos de grades agrícolas. STAB, v.14, p.1831, 1996a.
STOLF, R., SILVA, J. J. Metodologia de estimativa da potência do motor de tratores através do espaçamento, diâmetro e número de discos de grades agrícolas. In: CONGRESSO NACIONAL STAB, 6, 1996, Maceió. Anais... Maceió: STAB, 1996b. v.1. p.542-548. 